\title{
ARTIGOS
}

Submetido 11.03.2020. Aprovado 20.10.2020

Avaliado pelo sistema double blind review. Editora Científica: Claudia Araújo

Versão original | DOI: http://dx.doi.org/10.1590/So034-759020210604

\section{COMBINAÇÕES COMPORTAMENTAIS EXPLICATIVAS DA COLABORAÇÃO EM REDES DE SUPRIMENTOS}

\author{
Behavioral combinations that explain collaboration in the supply network \\ Combinaciones conductuales explicativas de la colaboración en redes de suministro
}

Roberta de Cássia Macedo ${ }^{1,2,3}$ | roberta.c.macedo@gmail.com | ORCID 0000-0002-5191-3542

Ricardo Silveira Martins' ${ }^{1}$ | ricardomartins.ufmg@gmail.com | ORCID 0000-0001-9717-3896

Jonathan Simões Freitas ${ }^{1}$ | jonathan.ufmg@gmail.com | ORCID o0oo-0001-5681-4327

\footnotetext{
Universidade Federal de Minas Gerais, Centro de Pós-Graduação e Pesquisas em Administração, Belo Horizonte, MG, Brasil

${ }^{2}$ Universidade FUMEC, Faculdade de Ciências Econômicas, Belo Horizonte, MG, Brasil

${ }^{3}$ Centro Universitário UNA, Belo Horizonte, MG, Brasil
}

\section{RESUMO}

O objetivo deste estudo foi investigar se combinações de fatores da Cultura Organizacional (CO) e dos Traços da Personalidade de gestores de compras e vendas são relevantes para explicar a colaboração na rede de suprimentos (CRS). Utilizaram-se como referenciais teóricos: Behavioral Operations Management (BOM), Comportamento Organizacional e Rede de Suprimentos (RS). Aplicou-se questionário eletrônico, autoadministrado e baseado em instrumentos difundidos na literatura. A análise foi feita pela Qualitative Comparative Analysis com condições dicotomizadas. A CRS com fornecedores apresentou diferentes associações com os construtos comportamentais quando comparados com a CRS com clientes, evidenciando que há diferenças comportamentais entre gestores de compras e de vendas. A qualificação da CRS sugere que as exigências comportamentais para a contratação de gestores de compras e de vendas são diferentes, assim como as relações com os clientes são prioritárias dentro da organização.

PALAVRAS-CHAVE | Traços da personalidade, cultura organizacional, csQCA, colaboração, rede de suprimentos.

\section{ABSTRACT}

The aim of this study was to investigate whether combinations of factors regarding organizational culture and the personality traits of purchase managers and sales managers are relevant to explain collaboration in the supply network (CSN). Our theoretical framework comprises Behavioral Operations Management, Organizational Behavior and Supply Network. We used a self-administered electronic questionnaire based on instruments that are well-known in the literature. We used Qualitative Comparative Analysis with dichotomized conditions. CSN with suppliers showed different associations with behavioral constructs compared to CSN with customers, which shows behavioral differences between purchase and sales managers. Our findings concerning CSN suggest that the behavioral requirements for hiring purchase and sales managers are different, and that customer relations are a priority within the organization.

KEYWORDS I Personality traits, organizational culture, cSQCA, collaboration, supply network.

\section{RESUMEN}

El objetivo de este estudio fue investigar si las combinaciones de factores de la cultura organizacional y de los rasgos de personalidad de los gerentes de compras y ventas son relevantes para explicar la colaboración en la cadena de suministro. Las referenciasteóricasson: BehavioralOperations Management, Comportamiento Organizacionaly Red deAbastecimiento. Se aplicó un cuestionario electrónico autoadministrado y basado en instrumentos difundidos en la literatura. El análisis se realizó mediante Qualitative Comparative Analysis con condiciones dicotomizadas. La colaboración en la cadena de suministro con los proveedores mostró diferentes asociaciones con las construcciones conductuales en comparación con la misma colaboración con los clientes, que demuestra diferencias de comportamiento entre los gerentes. De esta manera, la colaboración en la cadena de suministro sugiere que los requisitos conductuales para contratar gerentes de compras y ventas son diferentes, así como las relaciones con los clientes son una prioridad dentro de la organización.

PALABRAS CLAVE I Rasgos de personalidad, cultura organizacional, CSQCA, colaboración, red de abastecimiento. 


\section{INTRODUÇÃO}

Desde 1990 (Frohlich \& Westbrook, 2001), a gestão da rede de suprimentos (RS) tem gerado relacionamentos mais estreitos e intensos (interdependência) com fornecedores e clientes, para se alcançarem diferenciais diante dos concorrentes (Touboulic \& Walker, 2015). Atuando de maneira sincronizada, os parceiros da RS procuram responder melhor e de modo sustentável aos requisitos colocados pelo mercado (Barratt \& Barratt, 2011; Busse, Meinlschmidt, \& Foerstl, 2017).

Para acomodar interesses, muitas vezes contraditórios, estudos apontam que as RS de melhor performance são aquelas que conseguem promover relacionamentos colaborativos entre seus componentes. Cao, Vonderembse, Zhang, e Ragu-Nathan (2010) e Tsanos, Zografos e Harrison (2014) acreditam que a colaboração na rede de suprimentos (CRS) proporciona o aproveitamento dos recursos e conhecimentos de fornecedores e clientes.

Nesse escopo, ao integrar uma RS, as organizações gerenciam os relacionamentos intraorganizacionais e também os relacionamentos interorganizacionais (Flynn, Huo, \& Zhao, 2010; Wang, 2016). Porém, os relacionamentos em uma RS são conduzidos e praticados por indivíduos. Esses indivíduos estão imersos em elementos culturais que fazem parte do universo organizacional no qual estão inseridos, mas também possuem características próprias que orientam seus comportamentos e ações.

$\mathrm{Na}$ área de Operações, o campo que focaliza o indivíduo e o seu comportamento é o Behavioral Operations Management (BOM). O propósito do BOM é trazer as questões das pessoas ao campo de Operações (Katsikopoulos \& Gerd, 2013), por meio de uma interface com o Comportamento Organizacional e a Gestão de Recursos Humanos, abrangendo a psicologia de decisão individual e a influência da cultura nas interações entre os atores nesses processos (Loch \& Wu, 2007).

$\mathrm{Na}$ RS, entretanto, os indivíduos e a Cultura Organizacional (CO) têm sido pouco explorados, já que os estudos desse paradigma tendem a demonstrar as relações entre as organizações em uma perspectiva estruturalista e racionalista (Bendoly, Donohue, \& Schultz, 2006; Kim, Choi, Yan, \& Dooley, 2011; Siegler, Biazzin, \& Fernandes, 2014). Contudo, os indivíduos que estão nas organizações integrantes da RS personificam essas relações no nível micro, conferindo-lhes características afetivas e/ou profissionais. Assim, os indivíduos são elementos fundamentais para concretizar as relações colaborativas interorganizacionais (Tsanos et al., 2014), refletindo o comportamento de toda a rede (Galaskiewicz, 2011).

Já a CO influencia a maneira como os indivíduos agem e atuam no ambiente da organização, incluindo a RS (Makhdoom, Anjum, Kashif, \& Riaz, 2016). Nessa perspectiva, a CO pode atuar como facilitador ou dificultador na implementação de estratégias administrativas (Barbosa, 1996; Hilal, 2006; Lacerda, 2011; Souza \& Fenili, 2016), como a CRS.

Considerando, portanto, que a análise micro das relações, que é a compreensão do papel do indivíduo no contexto da RS, tem recebido moderada atenção da comunidade acadêmica na área de Operações, somada à importância do indivíduo e da CO em uma RS colaborativa, este estudo propõe-se a responder: De que modo os Traços da Personalidade e a CO se combinam para explicar a CRS?

0 restante do artigo está dividido em cinco seções. Na seção 2, discute-se o referencial teórico. Na seção 3, descreve-se a metodologia utilizada para coleta e análise dos dados. Na seção 4, os resultados são apresentados e discutidos. Na seção 5, apresentam-se as conclusões e recomendações para pesquisas futuras. 


\section{REFERENCIAL TEÓRICO}

\section{Behavioral Operations Management (BOM)}

Atualmente, pesquisas na área da Economia têm-se voltado para o comportamento humano, o que determinou a criação do campo Economia Comportamental (Thaler, 2016). Da mesma maneira, a área de Operações tem buscado ampliar os estudos sobre o indivíduo por meio do BOM. Os estudos seminais de Gino e Pisano (2007) visam incorporar o efeito do comportamento humano nas análises das atividades e relacionamentos no âmbito da logística e da gestão da RS (Gino \& Pisano, 2007; Siegler et al., 2014).

Essa valorização do comportamento do indivíduo vai além das soluções objetivas que as pesquisas em gestão da RS comumente abordam. Considera-se a realidade humana em que indivíduos apresentam-se com comportamentos irracionais e vulneráveis às influências do seu contexto social. Assim, há possibilidade de que esse comportamento "não controlado", difícil de prever e gerenciar, possa interferir positiva ou negativamente na gestão da RS ( Touboulic \& Walker, 2015).

Para o BOM, o comportamento do indivíduo reflete significativamente nos resultados alcançados na gestão da RS. Assim, vários pressupostos comumente empregados por modelos quantitativos desconsideram a variabilidade do comportamento do indivíduo. Alguns pressupostos consideram que os indivíduos: i) não são fator importante para a questão focal; ii) são deterministas e previsíveis; iii) não são afetados física ou psicologicamente por outros; iv) são imutáveis; v) não são parte do produto, serviço ou experiência do cliente; vi) não são afetados pelo orgulho, lealdade ou constrangimento (Boudreau et al., 2003).

\section{Colaboração na Rede de Suprimentos (CRS)}

A colaboração consiste em fornecedores e clientes trabalharem em conjunto para alcançar a melhoria mútua e contínua (Stank, Dittmann, \& Autry, 2011). A tomada de decisão conjunta é um fator colaborativo preponderante na RS (Ramanathan \& Gunasekaran, 2014).

Os níveis e os tipos de colaboração das organizações são afetados pela dependência, incerteza dos recursos e política econômica, além de surgirem no contexto de uma relação específica e desenvolverem-se por meio da interação contínua (Heide \& Miner, 1992). Para Touboulic e Walker (2015), a colaboração é uma estratégia indicada para a RS, entretanto não é implementada facilmente.

Para Busse et al. (2017), a colaboração é pautada na transparência e no compartilhamento de informações entre os parceiros da RS. Heide e Miner (1992) acreditam que é possível identificar a CRS por meio de quatro dimensões (Quadro 1).

\section{Quadro 1. Dimensões da colaboração}

\begin{tabular}{l|l}
\hline Dimensões & Significado \\
\hline Flexibilidade & Grau de acomodação das partes para se ajustar às necessidades dos parceiros. \\
\hline Compartilhamento de informações & Grau de disponibilização de informações que podem facilitar as atividades da outra parte. \\
\hline Resolução conjunta de problemas & $\begin{array}{l}\text { Grau de compartilhamento da responsabilidade pela manutenção das próprias relações e } \\
\text { pela busca conjunta de solução para os problemas. }\end{array}$ \\
\hline Restrição ao uso do poder & $\begin{array}{l}\text { Grau de renúncia da exploração de diferenciais de porte e dependência no } \\
\text { relacionamento, dada a oportunidade de o fazer. }\end{array}$ \\
\hline
\end{tabular}

Fonte: Heide e Miner (1992). 
A colaboração proporciona às organizações da RS a combinação das capacidades individuais. Assim, as organizações tornam-se complementares e criam valores que não conseguiriam de maneira independente (Barratt \& Barratt, 2011; Cao et al., 2010; Frohlich \& Westbrook, 2001; Touboulic \& Walker, 2015).

Segundo a literatura, quem colabora beneficia-se com vantagens mútuas, compartilhando recompensas, riscos e troca de informações (Ramanathan \& Gunasekaran, 2014), além de melhorar eficiência, eficácia e posicionamento de mercado (Tsanos et al., 2014), proporcionando vantagens superiores aos relacionamentos orientados à transação (Alfalla-Luque, Marin-Garcia, \& Medina-Lopez, 2015). Outros benefícios, como níveis mais elevados de confiança (Touboulic \& Walker, 2015) e comprometimento, retenção e aumento na carteira de clientes, e melhoria do desempenho, têm sido associados à CRS (Gligor \& Holcomb, 2013). Apesar dos benefícios, poucas organizações (como Honda e Toyota) têm demonstrado capacidade colaborativa como vantagem competitiva (Fawcett, McCarter, Fawcett, Webb, \& Magnan, 2015).

\section{Cultura Organizacional (CO)}

A CO tem sido amplamente estudada nas ciências sociais (Makhdoom et al., 2016), pois é uma variável relevante, atuando como dificultador ou facilitador na implementação de estratégias administrativas (Barbosa, 1996; Hilal, 2006; Lacerda, 2011; Souza \& Fenili, 2016). A CO aplica-se no coletivo e não individualmente, porém com efeitos no condicionamento do indivíduo (Lacerda, 2011; Oliven, 2009). A CO é o conjunto de crenças que direcionam os funcionários à compreensão das atitudes classificadas como aceitáveis e inaceitáveis na organização. Ela é transmitida por histórias e outros meios simbólicos, auxiliando o funcionário na compreensão do modus operandi da organização, enfatizando valores e normas para o seu comportamento (Griffin \& Moorhead, 2006; Lacerda, 2011; Robbins \& Judge, 2013).

A "cultura" são formas tácitas de perceber, pensar e reagir, sendo uma das forças mais poderosas e estáveis que operam nas organizações (Schein, 1996). Assim, a CO passa a fazer parte das pessoas e é aceita como verdadeira, exercendo uma poderosa influência sobre os funcionários (Fleury, 2009).

A CO, também definida como a programação coletiva da mente, faz com que os membros de um grupo sejam diferentes de outros grupos (Hofstede, Neuijen, Ohayv, \& Sanders, 1990). Para Souza e Felini (2016), o conceito de programação coletiva da mente equipara-se ao conceito de habitus proposto por Pierre Bourdieu. Os valores da CO podem ser compreendidos no contexto da organização em que foram gerados, não atingindo, necessariamente, outras organizações (Fleury, 2009; Makhdoom et al., 2016; Souza \& Fenili, 2016).

A teoria que define o construto CO foi desenvolvida por Hofstede et al. (1990) e utilizada para estudar a cultura em diversas organizações, e identificou duas dimensões da CO: prática e valores. A primeira dimensão compreende elementos que são visíveis para um observador: i) símbolos (cores, expressões verbais, gestos, quadros, entre outros); ii) heróis (indivíduos que possuem características admiradas pela cultura e servem de modelo de comportamento); iii) rituais (ações coletivas, hipoteticamente superficiais, mas essenciais para a cultura). A segunda dimensão é explicada como o modo como os significados culturais são percebidos pelas pessoas pertencentes à organização (Hofstede et al., 1990). 0 elemento “valores”, em sua maioria, é inconsciente e dificilmente discutido, porém é expresso por meio de comportamentos (Ferreira, Assmar, Estol, Helena, \& Cisne, 2002).

A partir dessas duas dimensões, Hofstede et al. (1990) desenvolveram um questionário e concluíram que os valores precisamente traçam a cultura, entretanto é por meio das práticas que a cultura influencia os membros da organização. 
Hilal (2006), Souza e Fenili (2016) apontam que há debates ontológicos e epistemológicos sobre a medição ou não da CO. Os estudiosos que sustentam a abordagem qualitativa defendem a descrição densa das organizações e acreditam que os casos são particulares, não sendo possível utilizar medidas padronizadas quantitativas, dificultando a replicação do estudo (Hilal, 2006). Por outro lado, a abordagem quantitativa possibilita que a metodologia seja aplicada em diferentes organizações, da mesma maneira, fornecendo uma base para comparações ou generalizações. Hofstede (um dos cientistas interculturais mais conhecidos) acredita que as abordagens devem ser complementares (Cooke \& Rousseau, 1988; Hilal, 2006).

\section{Traços da Personalidade}

A definição de personalidade mais utilizada é a elaborada por Gordon Allport há quase 70 anos. Segundo o autor, a personalidade é um sistema dinâmico dentro do indivíduo que determina seus ajustes para o ambiente. Assim, a personalidade é a soma total das maneiras pelas quais um indivíduo reage e interage com os outros (Robbins \& Judge, 2013). Para McCrae (2006), “[...] personalidade é o sistema no qual as tendências inatas da pessoa interagem com o ambiente social para produzir as ações e as experiências de uma vida individual” (p. 215).

O construto personalidade indica padrões de comportamento, atitudes e emoções que são típicos de um determinado indivíduo, diferenciando um do outro. Entretanto, esses traços apresentam alguma constância em uma pessoa e em diferentes contextos, bem como alguma estabilidade com o passar do tempo (Nakano, 2014). A personalidade é um agrupamento de características que são relativamente estáveis e diferenciam os indivíduos.

Os psicólogos identificaram vários Traços da Personalidade e diversas dimensões desses traços. Esses pesquisadores passaram a observar as correlações entre esses traços e suas dimensões e a agrupar os termos sinônimos. Como resultado, concluíram que há cinco grandes fatores (CGF) que abarcam os diversos termos dos Traços da Personalidade. Isso significa que “[...] quase todos os traços propostos por diferentes teorias da personalidade estavam relacionados a um ou a mais dos cinco fatores léxicos" (McCRae, 2006, p. 206).

O modelo dos CGF da personalidade, denominado Big Five, é um dos mais difundidos para descrever a estrutura da personalidade adulta e de maneira psicométrica (Nakano, 2014). É considerado uma teoria elucidativa da personalidade humana e possibilita uma descrição da personalidade de maneira simples, elegante e econômica (García, 2006; Silva \& Nakano, 2011). Os CGF originam-se da Teoria dos Traços da Personalidade e representam uma evolução conceitual e prática, pois especificam dimensões básicas da personalidade de maneira densa e replicável (Nakano, 2014).

Os CGF da personalidade podem ser aplicados nas organizações e são considerados relevantes para o entendimento do comportamento humano, e estão definidos e caracterizados no Quadro 2 (Bartholomeu, 2017; Faveri \& Knupp, 2018; Griffin \& Moorhead, 2006; Robbins \& Judge, 2013).

\section{Quadro 2. Cinco grandes fatores}

\begin{tabular}{l|l|l}
\hline Fator & Definição & Características \\
\hline Amabilidade & habilidade de relacionar-se bem com os outros & cooperação, compreensão e gentileza \\
\hline Consciência & $\begin{array}{l}\text { quantidade de objetivos em que cada indivíduo é capaz de } \\
\text { concentrar-se }\end{array}$ & $\begin{array}{l}\text { organização, reponsabilidade e } \\
\text { disciplina no trabalho }\end{array}$ \\
\hline Neuroticismo & alteração frequente de humor e sensibilidade emocional excessiva & ansiosos e preocupados \\
\hline Extroversão & conexão com o bem-estar sentido nos relacionamentos & amistosos e falantes \\
\hline Abertura & maleabilidade das crenças e dos interesses de uma pessoa a ouvir novas ideias
\end{tabular}

Fonte: Adaptado de John e Srivastava (1999), Bartholomeu (2017) e Faveri e Knupp (2018). 


\section{Variáveis e modelo teórico}

Esta pesquisa baseia-se na perspectiva do BOM e traz as questões das pessoas ao campo de Operações, por meio de uma interface com a CO e os Traços da Personalidade (Loch \& Wu, 2007).

A CO é representada pelos estudos de Hofstede et al. (1990) que foram utilizados em diversas organizações e países (Fleury, 2009). A personalidade foi tratada a partir do CGF (John \& Srivastava, 1999).

Hofstede et al. (1990) identificaram quatro elementos que foram distribuídos em dois grupos: prático, compreende os símbolos, heróis e rituais. "Valores", em sua maioria, é inconsciente e expresso por meio de comportamentos (Ferreira et al., 2002; Hofstede et al., 1990). Assim, a CO será tratada nas dimensões Cultura organizacional - Valores (Coval) e Cultura organizacional - Prática (Copra).

O CGF apresenta relevância baseada em sua aplicação em diversas amostras culturais, por possibilitarem a coleta de dados de maneira psicométrica (Bartholomeu, 2017; Faveri \& Knupp, 2018; Griffin \& Moorhead, 2006; Robbins \& Judge, 2013). Para John e Srivastava (1999), o CGF não representa uma visão reducionista da personalidade, pois cada dimensão resume um grande número de características distintas. Os CGF são: amabilidade (AMA), consciência (CONS), neuroticismo (NEU), extroversão (EXT) e abertura (ABE) (Quadro 2).

O modelo da pesquisa está na Figura 1, sua aplicação avalia as combinações das condições da Copra, Coval e os Traços da Personalidade - abertura, amabilidade, extroversão, consciência - para a CRS (outcome). 0 neuroticismo apresenta um sentido negativo, logo examinou-se o impacto da sua ausência na CRS.

\section{Figura 1. Modelo da pesquisa}




Analisaram-se as relações das organizações a montante, denominadas “Modelo Fornecedor", e a jusante, denominadas "Modelo Cliente" da RS. Ressalta-se que, apesar de a literatura afirmar que há uma relação de interdependência entre personalidade e CO (Oliven, 2009), este estudo pretende avaliar a combinação dessas variáveis para a CRS. Não há o objetivo de compreender e mensurar a causalidade mútua entre CO e personalidade.

\section{METODOLOGIA}

Realizou-se uma survey, cujas unidades de análise foram os relacionamentos na RS; as unidades de observação foram os gestores de compras e vendas.

\section{Amostra e coleta de dados}

A população desta pesquisa é considerada incalculável pelo significativo número de potenciais respondentes que estão alocados em diversas organizações (Malhotra, 2012). Foram utilizadas técnicas de amostragem não probabilísticas, sendo por acessibilidade ou conveniência (Hair, Babin, Money, \& Samouel, 2005).

0 tamanho da amostra foi calculado pelo G*Power 3.1.9.2. Como não há estudos anteriores que suportem essa escolha, selecionou-se o teste estatístico $f$ a um tamanho do efeito de o,15. 0 tamanho do efeito significa o quanto a variável independente influencia a variável dependente (Espírito-Santo \& Daniel, 2015). Assumiu-se um nível de significância de 5\% e um poder estatístico de 80\% (Hair, Hult, Ringle, \& Sarstedt, 2017); assim, o número de amostras necessárias para este estudo é de 68 respondentes.

0 instrumento de pesquisa passou por um pré-teste com aplicação presencial. Obtiveram-se 35 respondentes e procedeu-se com as adequações ao questionário e análise estatística dos dados para avaliação e definição do modelo. Parte da amostra final foi composta por respondentes do pré-teste, respondentes enquadrados completamente neste estudo.

O levantamento dos dados foi transversal e único (Malhotra, 2012) pelo SurveyMonkey ${ }^{\circledR}$. 0 questionário (autoadministrado) foi enviado entre 12/9/2017 e 31/7/2018 a uma base de 29.261 gestores (banco de dados dos pesquisadores e Núcleo de Pesquisas Logísticas - Nipelog). Obtiveram-se 611 respostas (2,09\%), sendo 452 incompletas. Para ampliar a amostra, o questionário foi disponibilizado no Facebook e Linkedln entre 23/1/2018 e 16/7/2018 e foi direcionado para o público-alvo desta pesquisa. Foram recebidas 65 respostas, sendo 50 incompletas.

Apesar de iniciar a coleta com uma base robusta e utilizar as redes sociais, a totalização da amostra (Tabela 1) representa menos de $1 \%$ da base inicial de contatos. Entretanto, o número de respondentes válidos está acima do tamanho da amostra calculada pelo GPower para os dois modelos.

\section{Tabela 1. Amostra válida}

\begin{tabular}{l|l|l|l|l}
\hline Modelo & E-mail & Redes Sociais & Pré-teste & Total \\
\hline Cliente & 91 & 2 & 13 & 106 \\
\hline Fornecedor & 68 & 13 & 11 & 92 \\
\hline
\end{tabular}




\section{Instrumento de pesquisa}

Utilizaram-se questionários validados e difundidos na literatura (Quadro 3).

Quadro 3. Instrumentos da pesquisa

\begin{tabular}{l|c|c}
\hline Construto & Teoria & Instrumento \\
\hline Personalidade & Big Five & BFI-44 \\
\hline Cultura Organizacional & Comportamento Organizacional & IBACO (reduzido) \\
\hline Colaboração na RS & Paradigma da RS & Modelo de Heide e Miner \\
\hline
\end{tabular}

O Big Five Inventory (BF1-44) foi utilizado porque, diante dos diversos instrumentos estabelecidos, ele não consta na lista do Sistema de Avaliação de Testes Psicológicos (Satepsi) como um instrumento com aplicação obrigatória por psicólogos (Satepsi, 2016). Adicionalmente, é curto, eficiente e de fácil entendimento (John \& Srivastava, 1999).

Para avaliar a CO, utilizou-se o Ibaco, que “[...] representa um esforço de construção de um instrumento originalmente nacional destinado a avaliar a cultura organizacional por meio de seus valores e suas práticas” (Ferreira \& Assmar, 2008, p. 128).

A CRS foi trabalhada a partir das definições de Heide e Meiner (1992), pois é um dos estudos mais referenciados por pesquisadores do relacionamento comprador-fornecedor, sendo citado em mais de 800 estudos (Brito, Sambiase, Ferreira, \& Silva, 2017).

Quanto ao questionário, utilizaram-se perguntas fechadas e escala Likert de seis pontos; o número par foi escolhido porque elimina o "ponto do meio". Esse ponto pode proporcionar uma fuga fácil, e há evidências de que alguns indivíduos que optam por pontuações neutras não se consideram necessariamente neutros em relação ao objeto avaliado (Matell \& Jacoby, 1971).

Para medir detalhadamente a percepção do respondente, foi utilizada a barra deslizante, que proporciona a quantificação decimal. Devido a essa escolha, os números não são usados, evitando tendências para seguir a mesma marcação (Stone, Bleibaum, \& Thomas, 2012). A escala partiu de “discordo totalmente" para "concordo totalmente”.

\section{Qualitative Comparative Analysis (QCA)}

O QCA é um método desenvolvido por Charles Ragin, nos anos de 1980, em um estudo comparativo de casos utilizando álgebra booleana e teoria de conjuntos (Marx, Cambre, \& Rihoux, 2013). O QCA objetiva integrar os melhores recursos da abordagem orientada a casos com a abordagem orientada a variáveis. Considera-se um "caso" cada gestor respondente da survey (Marx et al., 2013; Rihoux \& Ragin, 2009).

Para Fiss (2007), o QCA é um método configuracional qualitativo, que procura compreender como as causas combinam-se para criar resultados. Para Marx (2010), o QCA permite comparar sistematicamente diferenças e semelhanças de configurações das condições em um conjunto de casos, permitindo aos pesquisadores explorar os dados e desenvolver modelos explicativos em termos de dependências booleanas. Assim, o QCA distingue os fatores necessários e/ou suficientes para a ocorrência do resultado (Blackman, 2013).

O QCA é capaz de lidar com níveis consideráveis de complexidade causal enquanto retém a qualidade holística do fenômeno em estudo (Fiss, 2007). Foi originalmente projetado para lidar com pequenas amostras, mas é aplicado com sucesso à análise de grandes bancos de dados (Fiss, 2007; Marx, 2010; Rihoux \& Ragin, 2009). 
Escolheu-se o QCA, pois, conforme Blackman (2013), a realidade é complexa para ser explicada por relacionamentos bi ou multivariados baseados na soma do efeito líquido de cada variável independente. Abordagens configuracionais, como o QCA, consideram que o resultado (outcome) é causado por diferentes combinações lógicas das condições, as quais não exercem o seu poder causal isoladamente (Fiss, 2007). Assim, em vez de analisar a relação entre uma variável que depende de outras variáveis que não interagem entre si, o QCA compara casos empíricos concretos, vistos como configurações lógicas de condições explicativas e associados à presença ou ausência de um resultado (Marx, 2010).

Utilizou-se o crisp set QCA (csQCA), que trabalha com condições dicotomizadas. No csQCA, as variáveis assumem dois valores: o (falso) ou 1 (verdadeiro). Essa simplificação binomial significa uma perda de informação, dado que as variáveis originais são contínuas. Entretanto, Blackman (2013) preconiza que os dados dicotomizados permitem analisar se determinada prática ou condição contextual é relevante para a ocorrência ou não do resultado. A quantidade de casos deste estudo suporta o csQCA, pois possibilita uma consistência maior, respeitando os limites de especificação desse tipo de modelo (Marx \& Dusa, 2010).

Uma consideração importante no csQCA é como decidir os pontos de quebra para codificação das condições, em sistema binário, a partir das medidas originais em escalas contínuas (Blackman, 2013). Essa decisão é uma interpretação, refletindo o "qualitativo" do QCA. Para dicotomizar os dados, considerou-se o ponto de corte de 3,50 da escala Likert (acima da média de seis pontos). Quanto maior a concordância dos respondentes, maior a presença dos construtos medidos. Portanto, valores iguais ou acima de 3,50 significam a presença da variável, e os valores abaixo significam a ausência da variável em cada item e para cada caso.

\section{RESULTADOS}

Fez-se uma análise descritiva da amostra na perspectiva dos setores econômicos e porte das organizações em que os respondentes trabalham (Tabela 2). Nos dois modelos, o setor de serviços é o mais representativo. A renda anual das organizações foi classificada conforme a receita operacional bruta anual, definida pelo Banco Nacional de Desenvolvimento Econômico e Social (BNDES).

\section{Tabela 2. Análise descritiva}

\begin{tabular}{|c|c|c|c|}
\hline & & Cliente & Fornecedor \\
\hline \multirow{4}{*}{$\begin{array}{l}\text { Setores } \\
\text { Econômicos }\end{array}$} & Serviço & $39 \%$ & $46 \%$ \\
\hline & Varejo & $28 \%$ & $29 \%$ \\
\hline & Indústria & $25 \%$ & $22 \%$ \\
\hline & Atacado & $8 \%$ & $3 \%$ \\
\hline \multirow{5}{*}{$\begin{array}{l}\text { Porte das } \\
\text { Organizações }\end{array}$} & Grande (maior que R\$ 300 milhões) & $16 \%$ & $12 \%$ \\
\hline & Média (de R\$ 3,6 milhões até R\$ 300 milhões) & $36 \%$ & $35 \%$ \\
\hline & Pequena (de R\$ 360 mil até R\$ 3,6 milhões) & $25 \%$ & $27 \%$ \\
\hline & Microempresa (até R\$360 mil) & $23 \%$ & $22 \%$ \\
\hline & Sem fins lucrativos & - & $4 \%$ \\
\hline
\end{tabular}


A tabela-verdade é o resultado que apresenta as configurações lógicas possíveis de atributos causais para formar os conjuntos parcimoniosos explicativos da variável dependente (Rihoux \& Meur, 2009). Nessa primeira análise, a tabela-verdade conteve os resultados "1" "o" e apresentou contradições (i.e. configurações lógicas que se verificaram tanto em casos de colaboração quanto de não colaboração). Foi necessário resolver as contradições. Uma maneira para solucionar as contradições é adicionando novas condições (Schneider \& Wagemann, 2010). Contudo, como a base de dados original foi a survey, optou-se por relaxar a restrição de consistência absoluta de cada configuração (Ragin, 2000; Schneider \& Wagemann, 2010). Assim, a consistência das configurações foi reduzida para 0,75 , como sugere Ragin (2000).

Verificou-se na tabela-verdade, para os dois modelos em análise, que nenhuma das condições isoladas é suficiente ou necessária para o resultado. Na sequência, analisou-se a combinação das condições (conjunções) por meio da consistência e da cobertura.

As configurações explicativas simplificadas, para os dois modelos, foram geradas pela minimização da solução conservadora pelo método melhorado do Quine-McCluskey no pacote QCA, do software R® (Duşa, 2007). Essas expressões lógicas representam as possibilidades alternativas de explicação do resultado, desconsiderando, no processo de simplificação, todas as configurações originais para as quais não se tenha verificado casos empíricos correspondentes. As Tabelas 3 e 4 mostram a minimização desses conjuntos a partir da lógica booleana para os Modelos Cliente e Fornecedor, respectivamente.

A coluna "configurações" mostra as conjunções causais associadas à presença da CRS. Na sequência, a medida de consistência (principal critério de validação do QCA) indica se a conjunção é satisfatoriamente suficiente (> que 0,75). A medida de cobertura apresenta a quantificação da relevância empírica da combinação causal, em termos do percentual de casos de colaboração cobertos pela respectiva configuração. A medida de cobertura única apresenta quantos casos, em termos percentuais, são cobertos somente por aquela configuração (Betarelli \& Ferreira, 2018).

Tabela 3. Configurações parcimoniosas: Modelo Cliente

\begin{tabular}{|c|c|c|c|c|}
\hline & Configurações & Consistência & Cobertura & Cobertura Única \\
\hline 1 & $\mathrm{COPRA}^{\star}$ COVAL & 0.940 & 0.741 & 0.294 \\
\hline 2 & abe ${ }^{\star}$ cons $^{\star}$ EXT $^{\star}$ COPRA & 1.000 & 0.035 & 0.012 \\
\hline 3 & ama*ABE*CONS*neu*EXT & 1.000 & 0.035 & 0.012 \\
\hline 4 & ama*ABE*copra & 1.000 & 0.012 & 0.000 \\
\hline 5 & ama*CONS*copra & 1.000 & 0.012 & 0.000 \\
\hline 6 & ama*NEU*copra & 1.000 & 0.012 & 0.000 \\
\hline 7 & $\mathrm{AMA}^{\star}{ }^{*}$ bbe neu & 1.000 & 0.047 & 0.000 \\
\hline 8 & $\mathrm{AMA}^{\star} \mathrm{abe}^{\star}$ COPRA & 1.000 & 0.153 & 0.000 \\
\hline 9 & $\mathrm{AMA}^{\star}$ cons $^{*}$ neu & 1.000 & 0.047 & 0.000 \\
\hline 10 & $\mathrm{AMA}^{\star}$ cons $^{\star} \mathrm{EXT}$ & 1.000 & 0.059 & 0.000 \\
\hline 11 & $\mathrm{AMA}^{*} \mathrm{CONS}^{*} \mathrm{NEU}^{*} \mathrm{COPRA}$ & 0.950 & 0.447 & 0.000 \\
\hline 12 & $\mathrm{AMA}^{\star} \mathrm{NEU}^{\star} \mathrm{EXT}^{\star}{ }^{\star} \mathrm{COPRA}$ & 0.953 & 0.482 & 0.000 \\
\hline
\end{tabular}

Nota. Letra maiúscula =1; Letra minúscula =0; * = operador lógico “E”; + = operador lógico "OU. 
A Tabela 3 mostra 12 caminhos que levam ao mesmo resultado. Com base na consistência, todas essas conjunções são suficientes ( $>$ que 0,75 ) para a presença da CRS. Entretanto, individualmente, as conjunções não são necessárias para a CRS. Como todas as conjunções são suficientes, fez-se uma análise das conjunções que apresentam maior cobertura (conjunções 1, 11 e 12). Quanto às conjunções 2 e 3, apesar de possuírem percentual de cobertura única acima de zero, considerou-se que esse valor representa um outlier, já que corresponde a um caso da amostra. Outro ponto é que a seta representada nas conjunções indica implicação lógica e, portanto, que o termo à sua esquerda é suficiente para o termo à direita.

\section{COPRA*COVAL + \\ $\mathrm{AMA}{ }^{*} \mathrm{NEU}{ }^{\star} \mathrm{EXT}{ }^{*} \mathrm{COPRA}+$ \\ $A M A{ }^{*} \mathrm{CONS}^{\star} N E{ }^{*}{ }^{*} \mathrm{COPRA} \rightarrow \mathrm{CRS}$ CLIENTE}

A expressão lógica que simplifica as três conjunções selecionadas é:

COPRA* $(C O V A L+(A M A * N E U *(E X T+C O N S)) \rightarrow$ CRS CLIENTE

A expressão lógica para o Modelo Cliente apresenta um caminho simples e um caminho complexo, e a condição Copra está presente nos dois caminhos. Logo, Copra é um fator comum, causalmente relevante para a CRS.

O caminho simples combina Copra com Coval e demonstra que as dimensões da CO, quando combinadas, confirmam sua atuação facilitadora para a CRS. No caminho complexo, Copra combina com algum traço da personalidade para alcançar o resultado. Assim, a combinação entre Copra com a facilidade de relacionamento e a estabilidade emocional do vendedor resulta na CRS. Semelhantemente, no caminho complexo, a combinação entre a Copra com características afetuosas e estáveis do vendedor, além de organizado e orientado para objetivos, também resulta na CRS.

Tabela 4. Configurações parcimoniosas: Modelo Fornecedor

\begin{tabular}{c|l|c|c|c}
\hline & Configurações & Consistência & Cobertura & Cobertura Única \\
\hline 1 & ABE*NEU*COVAL & 0.919 & 0.567 & 0.067 \\
\hline 2 & abe*CONS*NEU*EXT*coval & 1.000 & 0.067 & 0.033 \\
\hline 3 & AMA*COVAL $^{\star}$ & 0.923 & 0.600 & 0.000 \\
\hline 4 & AMA*abe*NEU $^{\star}$ & 0.875 & 0.117 & 0.000 \\
\hline 6 & AMA*ABE*neu*EXT $^{*}$ & 1.000 & 0.033 & 0.000 \\
\hline
\end{tabular}

O Modelo Fornecedor (Tabela 4) apresentou seis caminhos que levam ao resultado. Como procedeu no Modelo Cliente, com base na consistência, todas as conjunções são suficientes para a presença da CRS. Como todas as conjunções são suficientes, também foi feita uma análise das conjunções que apresentam maior cobertura (itens 1 e 3). Quanto à conjunção 2, apesar de possuir percentual de cobertura única acima de zero, considerou-se que esse valor representa um outlier, já que corresponde a três casos da amostra. 


\section{$\mathrm{ABE}{ }^{*} \mathrm{NEU} \mathrm{COVVAL}^{*}$ $\mathrm{AMA}^{\star}$ COVAL $\rightarrow$ CRS FORNECEDOR}

A expressão lógica que simplifica as duas conjunções selecionadas é: $\mathrm{COVAL}^{\star}\left(\mathrm{ABE}{ }^{\star} N E U+\mathrm{AMA}\right) \rightarrow$ CRS FORNECEDOR

A expressão lógica para o Modelo Fornecedor apresenta um caminho simples e um caminho complexo, e a condição Coval está presente nos dois caminhos. Logo, Coval é uma causa comum para a CRS. Ademais, para resultar na CRS, essa condição precisa estar combinada com a abertura, neuroticismo ou amabilidade.

O caminho simples combina Coval com AMA e demonstra que o modo como os significados culturais são percebidos pelos compradores, associado à sua capacidade de se relacionar bem, resulta na CRS. Já no caminho complexo, Coval combina com ABE e NEU para alcançar o resultado. Esse caminho complexo demonstra que o modo como os significados culturais são percebidos pelos compradores, associado a uma característica flexível e emocionalmente estável, também conduz à CRS.

\section{Discussão dos resultados}

Os resultados dos modelos demonstram que as condições inicialmente previstas precisam combinar-se para explicarem o resultado (Fiss, 2007). Mais especificamente, Copra está sempre presente nas conjunções analisadas para o Modelo Cliente e Coval, para o Modelo Fornecedor. Essa presença mais relevante de cada dimensão em cada modelo pode ser explicada pelo fato de que Copra compreende elementos que são visíveis para um observador, logo as organizações buscam enfatizar, por meio de símbolos, heróis (i.e. vendedor do mês) e rituais, a importância dos relacionamentos com os clientes (Ferreira et al., 2002). Como as relações com os fornecedores não utilizam os elementos visíveis para um observador, os compradores percebem a CO por meio da dimensão Coval, que, em sua maioria, é inconsciente e dificilmente discutida (Ferreira et al., 2002; Hofstede et al., 1990).

Contudo, Copra e Coval não garantem o resultado sozinhas, e sim por meio de caminhos alternativos. Assim, evidencia-se que, apesar de a CO aplicar-se ao coletivo, seus efeitos discorrem sobre o condicionamento do indivíduo (Lacerda, 2011; Oliven, 2009). Adicionalmente, há a possibilidade de gerar a CRS mesmo quando Copra e Coval estão ausentes.

Os resultados mostram que as organizações tratam de modo diferente seus relacionamentos com clientes e com fornecedores, e que os gestores de vendas ou compras que colaboram na RS possuem diferentes Traços da Personalidade. Essa constatação corrobora Nakano (2014), que afirmou que os traços apresentam alguma constância em uma pessoa e em diferentes contextos.

A CO também causa influências diferentes nos modelos, sendo sua influência no Modelo Cliente preponderante comparada ao Modelo Fornecedor. Isso porque, no Modelo Cliente, as dimensões Copra e Coval, quando combinadas, são suficientes para alcançar o resultado. No Modelo Fornecedor, a dimensão Coval está combinada com algum traço da personalidade para causar o outcome.

Os resultados também demonstram que, ao considerar que a personalidade determina seus ajustes para o ambiente (Robbins \& Judge, 2013), os Traços da Personalidade do gestor de compras e vendas trazem interferências diferentes para a CRS. Isso porque os Traços da Personalidade aparecem nos dois modelos; contudo, no Modelo Fornecedor, para que haja o resultado esperado, uma das suas dimensões sempre estava presente na conjunção, combinando com Cova, diferentemente do Modelo Cliente, que possui uma conjunção em que a CO é suficiente para conduzir ao resultado. 
Essas diferenças comportamentais podem ser importantes, já que o vendedor focaliza sua ação no atingimento de metas de faturamento e lucro. 0 comprador, por sua vez, é pressionado a negociar melhores condições (e.g. preço, prazo de entrega e pagamento, qualidade) para a organização. Assim, a presença da CO pode estar direcionada para o fortalecimento das relações, com os clientes promovendo a programação coletiva da mente dos membros desse grupo, tornando os gestores de vendas diferentes dos gestores de compras (Hofstede et al., 1990).

Griffin e Moorhead (2006) asseguram que os Traços da Personalidade do indivíduo direcionam suas percepções e ações. Contudo, apesar de os traços propostos por diferentes teorias da personalidade estarem relacionados a um ou a mais dos cinco fatores léxicos (McCrae, 2006), os traços não comandam isoladamente as decisões dos indivíduos, em função de sua forma de agir mediante uma situação ser o resultado dos Traços da Personalidade e de sua inter-relação com a CO.

A constatação da importância da combinação da CO e do indivíduo para a CRS contribui para a ampliação e o fortalecimento do BOM (Tsanos et al., 2014), demonstrando que o comportamento humano e o organizacional são influenciadores da CRS (Tatham, Wu, Kovács, \& Butcher, 2017). Assim, a capacidade da organização em administrar e integrar a rede de relações interorganizacionais está intrincada com o comportamento dos indivíduos que protagonizam essa relação (Stank et al., 2011).

\section{CONCLUSÃO}

Este artigo apresenta a análise comportamental dos relacionamentos interorganizacionais na RS de 198 organizações localizadas em Minas Gerais, sendo 106 respondentes gestores de vendas e 92 gestores de compras. A coleta dos dados sobre como o comportamento humano e o organizacional se combinam para explicar a CRS foi feita utilizando os construtos: Traços da Personalidade, CO e CRS.

Os resultados revelam diferentes combinações entre os Traços da Personalidade e a CO para resultar na CRS. Essas diferenças combinatórias são evidenciadas entre os Modelos Cliente e Fornecedor, mas também dentro de cada modelo. Os resultados mostraram que há uma programação coletiva da mente direcionada para os relacionamentos com os clientes e outra para os relacionamentos com os fornecedores. Ademais, apesar da personificação das relações interorganizacionais, a CO sobressai aos Traços da Personalidade no Modelo Cliente.

\section{Contribuição teórica e gerencial}

Na perspectiva teórica, buscou-se ampliar os estudos sobre o comportamento humano no campo de Operações (Loch $\& W u, 2007)$. Assumindo que os relacionamentos na RS são conduzidos e praticados por indivíduos, os resultados demonstraram que, nos relacionamentos com os clientes, a CO exerce poderosa influência nos gestores, pois orienta suas ações. Assim, tratando diferentemente os relacionamentos com clientes e com fornecedores, criam-se diferenças comportamentais entre os gestores de compras e vendas, incluindo os atributos dos Traços da Personalidade.

Além disso, as comparações dos resultados com a literatura indicam a CO como forte atributo de impacto nas relações com os clientes. Já na relação com os fornecedores, a CO é percebida, mas a sua prática e manifestação não é preponderante, pois a sua presença está combinada com algum traço da personalidade para a CRS. Entretanto, nos dois modelos, o indivíduo provoca um impacto comportamental na CRS e apresenta-se como elemento essencial, seja como replicador da CO, seja como potencializador dos negócios, por suas características pessoais. Finalmente, compreende-se que este estudo traz contribuições para a ampliação das pesquisas em BOM. 
Na perspectiva gerencial, a análise traz contribuições práticas, pois focaliza o indivíduo como unidade de observação na RS (Katsikopoulos \& Gerd, 2013). Também estimula as organizações a considerarem as relações micro no direcionamento de esforços para o engajamento colaborativo na RS.

O estudo revela, no âmbito dos Traços da Personalidade, a importância em considerar, nas contratações dos gestores de compras e vendas, as atribuições comportamentais (que são diferentes entre eles), contribuindo para o valor estratégico da CRS.

\section{Limitações e estudos futuros}

Como limitação, este estudo analisa o tema de maneira agregada, desconsiderando qualidades específicas das organizações como especificidades das relações com clientes e fornecedores, mercado de atuação e desempenho financeiro. Dessa forma, conforme sugerido por Hilal (2006), para vencer essas limitações, propõe-se o aprofundamento na análise de casos.

Recomenda-se, para pesquisas futuras, investigar se as características comportamentais melhoram o desempenho financeiro da organização na RS e se a CO e os Traços da Personalidade influenciam a confiança nos relacionamentos na RS.

\section{REFERÊNCIAS}

Alfalla-Luque, R., Marin-Garcia, J. A., \& Medina-Lopez, C. (2015). An analysis of the direct and mediated effects of employee commitment and supply chain integration on organisational performance. International Journal of Production Economics, 162, 242-257. doi: 10.1016/j.ijpe.2014.07.004

Barbosa, L. N. de H. (1996). Cultura administrativa: Uma nova perspectiva das relações entre antropologia e administração. RAE-Revista de Administração de Empresas, 36(4), 6-19. doi: 10.1590/So034-75901996000400002

Barratt, M., \& Barratt, R. (2011). Exploring internal and external supply chain linkages: Evidence from the field. Journal of Operations Management, 29(5), 514-528. doi: 10.1016/j. jom.2010.11.006

Bartholomeu, D. B. (2017). Traços de personalidade e comportamentos de risco no trânsito: Um estudo correlacional. Psicologia Argumento, 26(54), 193. doi: 10.7213/psicolargum. v26i54.19685

Bendoly, E., Donohue, K., \& Schultz, K. L. (2006). Behavior in operations management: Assessing recent findings and revisiting old assumptions. Journal of Operations Management, 24(6), 737-752. doi: 10.1016/j.jom.2005.10.001

Betarelli, A. A., Junior, \& Ferreira, S. F. (2018). Introdução à análise qualitativa comparativa e aos Conjuntos Fuzzy (Fsqca). Recuperado de http://repositorio.enap.gov.br/ bitstream/1/3333/1/Livro_Introdução à análise qualitativa comparativa e aos conjuntos Fuzzy.pdf

Blackman, T. (2013). Exploring explanations for local reductions in teenage pregnancy rates in England: An approach using qualitative comparative analysis. Social Policy and Society, 12(1), 61-72. doi: 10.1017/S1474746412000358
Boudreau, J., Boudreau, J., Hopp, W., Hopp, W., McClain, J. O., McClain, J. O., ... Thomas, L. J. (2003). Commissioned paper: On the interface between operations and human resources management. Manufacturing \& Service Operations Management, 5(3), 179-202. doi: 10.1287/ msom.5.3.179.16032

Brito, E. P. Z., Sambiase, M. F., Ferreira, F. C. M., \& Silva, A. A. Da. (2017). The effect of uncertainty and cooperative behavior on operational performance: Evidence from Brazilian firms. Journal of Operations and Supply Chain Management, 10(2), 71. doi: $10.12660 /$ joscmv1on2p71-84

Busse, C., Meinlschmidt, J., \& Foerstl, K. (2017). Managing information processing needs in global supply chains: A prerequisite to sustainable supply chain management. Journal of Supply Chain Management, 53(1), 87-113. doi: 10.1111/jscm.12129

Cao, M., Vonderembse, M., Zhang, Q., \& Ragu-Nathan, T. S. (2010). Supply chain collaboration: Conceptualisation and instrument development. International Journal of Production Research, 48(22), 6613-6635. doi: 10.1080/00207540903349039

Cooke, R. A., \& Rousseau, D. M. (1988). Behavioral norms and expectations: A quantitative approach to the assessment of organizational culture. Group \& Organization Management, 13(3), 245-273. doi: 10.1177/105960118801300302

Duşa, A. (2007). User manual for the QCA(GUI) package in R. Journal of Business Research, 60(5), 576-586. doi: 10.1016/j. jbusres.2007.01.002 
Espírito-Santo, H., \& Daniel, F. (2015). Calcular e apresentar tamanhos do efeito em trabalhos científicos: As limitações do p 0,05 na análise de diferenças de médias de dois grupos. Revista Portuguesa de Investigação Comportamental e Social, 1(1), 3-16. doi: 10.5965/1984723816352016153

Faveri, D. B. De, \& Knupp, P. D. S. (2018). Finanças comportamentais: Relação entre traços de personalidade e vieses comportamentais. Base - Revista de Administração e Contabilidade, 15(1), 18-30. doi: 10.4013/base.2018.151.02

Fawcett, S. E., McCarter, M. W., Fawcett, A. M., Webb, G. S., \& Magnan, G. (2015). Supply chain management: An international journal article information. Supply Chain Management: An International Journal, 20(6), 648-663. doi: 10.1108/SCM-08-2015-0331

Ferreira, M. C., \& Assmar, E. M. L. (2008). Cultura organizacional. In M. M. M. Siqueira (Eds.), Medidas do comportamento organizacional: Ferramentas de diagnóstico e de gestão (pp. 125-138). Porto Alegre, RS: Artmed.

Ferreira, M. C., Assmar, E. M. L., Estol, K. M. F., Helena, M. C. da C. C., \& Cisne, M. do C. F. (2002). Desenvolvimento de um instrumento brasileiro para avaliação da cultura organizacional. Estudos de Psicologia, 7(2), 271-280. doi: 10.1590/S1413-294X2002000200008

Fiss, P. C. (2007). A set-theoretic approach to organizational configurations. Academy of Management Review, 32(4), 11801198. doi: 10.5465/AMR.2007.26586092

Fleury, M. T. L. (2009). Organizational culture and the renewal of competences. BAR - Brazilian Administration Review, 6(1), 1-14. doi: 10.1590/S1807-76922009000100002

Flynn, B. B., Huo, B., \& Zhao, X. (2010). The impact of supply chain integration on performance: A contingency and configuration approach. Journal of Operations Management, 28(1), 58-71. doi: 10.1016/j.jom.2009.06.001

Frohlich, M. T., \& Westbrook, R. (2001). Arcs of integration: An international study of supply chain strategies. Journal of Operations Management, 19(2), 185-200. doi: 10.1016/ So272-6963(00)00055-3

Galaskiewicz, J. (2011). Studying supply chains from a social network perspective. Journal of Supply Chain Management, 47(1), 4-8. doi: 10.1111/j.1745-493X.2010.03209.x

García, L. F. (2006). Teorias psicométricas da personalidade. In C. F. Mendonza, R. Colom,(Eds.), Introdução à psicologia das diferenças individuais (pp. 219-242). Porto Alegre, RS: Artmed

Gino, F., \& Pisano, G. (2007). Toward a theory of behavioral operations. Manufacturing \& Service Operations Management, 10(4), 676-691. doi: 10.1287/msom.1070.0205

Gligor, D. M., \& Holcomb, M. (2013). The role of personal relationships in supply chains: An exploration of buyers and suppliers of logistics services. The International Journal of Logistics Management, 24(3), 328-355. doi: 10.1108/IJLM-072012-0067

Griffin, R. W., \& Moorhead, G. (2006). Fundamentos do comportamento organizacional. São Paulo, SP: Ática.
Hair, J. F. J., Babin, B., Money, A. H., \& Samouel, P. (2005). Fundamentos de métodos de pesquisa em administração. Porto Alegre, RS: Bookman.

Hair, J. F. J., Hult, G. T. M., Ringle, C. M., \& Sarstedt, M. (2017). A primer on partial least squares structural equation modeling (PLS-SEM). Los Angeles, USA: Sage Publications.

Heide, J. B., \& Miner, A. S. (1992). The shadow of the future: Effects of anticipated interaction and frequency of contact on buyer-seller cooperation. Academy of Management Journal, 35(2), 265-291. doi: 10.2307/256374

Hilal, A. V. G. De. (2006). Brazilian national culture, organizational culture and cultural agreement: Findings from a multinational company. International Journal of Cross Cultural Management, 6(2), 139-167. doi: 10.1177/1470595806066325

Hofstede, G., Neuijen, B., Ohayv, D. D., \& Sanders, G. (1990). Measuring organizational cultures: A qualitative and quantitative study across twenty cases. Administrative Science Quarteriy, 35(2), 286-316. doi: 10.2307/2393392

John, O. P., \& Srivastava, S. (1999). The Big-Five trait taxonomy: History, measurement, and theoretical perspectives. In L. A. Pervin \& O. P. John (Eds.), Handbook of personality: Theory and research (pp. 102-138). New York: Guilford Press. doi: citeulike-article-id:3488537

Katsikopoulos, K. V., \& Gerd, G. (2013). Behavioral operations management: A blind spot and a research program. 49(1), 3-7. doi: 10.1111/j.1745-493x.2012.03285.x

Kim, Y., Choi, T. Y., Yan, T., \& Dooley, K. (2011). Structural investigation of supply networks: A social network analysis approach. Journal of Operations Management, 29(3), 194-211. doi: 10.1016/j.jom.2010.11.001

Lacerda, D. P. (2011). Cultura organizacional: Sinergias e alergias entre Hofstede e Trompenaars. Revista de Administração Pública, 45(5), 1285-1301. doi: 10.1590/So03476122011000500003

Loch, C. H., \& Wu, Y. (2007). Behavioral operations management. Journal of Experimental Psychology: General, 136(1), 23-42.

Makhdoom, H. ur R., Anjum, A., Kashif, M. T., \& Riaz, W. (2016). Supply chain integration and operational performance: Moderating role of organizational culture. International Journal of Academic Research in Business and Social Sciences, 6(12), 644-657. doi: 10.6007/IJARBSS/v6-i12/2526

Malhotra, N. (2012). Pesquisas de marketing: Uma orientação aplicada (6a ed.). Porto Alegre, RS: Artmed.

Marx, A. (2010). Crisp-set qualitative comparative analysis (csQCA) and model specification: Benchmarks for future CsQCA applications. International Journal of Multiple Research Approaches, 4(2), 138-158. doi: 10.5172/mra.2010.4.2.138

Marx, A., Cambre, B., \& Rihoux, B. (2013). CRISP-SET qualitative comparative analysis in organizational studies. In P. Fiss, B. Cambré, \& A. Marx (Eds.), Research in the Sociology of Organizations: Configurational Theory and Methods in Organizational Research (Vol. 38, Issue January, pp. 23-47). Bingley (UK): Emerald Group Publishing. doi: 10.1108/S0733558X(2013)0000038006 
Marx, A., \& Dusa, A. (2010). Crisp-set qualitative comparative analysis (CSQCA) and model specification: Benchmarks for future CSQCA applications. International Journal of Multiple Research Approaches, 4(2), 138-158. https://doi.org/10.5172/ mra.2010.4.2.138

Matell, M. S., \& Jacoby, J. (1971). Is there an optimal number of alternatives for Likert scale items? Study I: Reliability and validity. Educational and Psychological Measurement, 31(3), 657-674. doi: 10.1177/001316447103100307

McCrae, R. R. (2006). O que é personalidade. In C. E. FloresMendoza, R. Colom (Eds.), Introdução à psicologia das diferenças individuais (pp. 203-218). Porto Alegre, RS: Artmed.

Nakano, T. de C. (2014). Personalidade: Estudo comparativo entre dois instrumentos de avaliação. Estudos de Psicologia, 31(3), 347-357. doi: 10.1590/0103-166X2014000300003

Oliven, R. G. (2009). Cultura e personalidade. In R. G. Oliven (Ed.), Metabolismo social da cidade e outros ensaios (p. 51). Centro Edelstein de Pesquisas Sociais. Recuperado de https://core. ac.uk/reader/205762230

Ragin, C. C. (2000). Fuzzy-set social science. Chicago, USA: University of Chicago Press.

Ramanathan, U., \& Gunasekaran, A. (2014). Supply chain collaboration: Impact of success in long-term partnerships. International Journal of Production Economics, 147(Part B), 252-259. doi: 10.1016/j.ijpe.2012.06.002

Rihoux, B., \& Meur, G. De. (2009). Crisp-set qualitative comparative analysis (cSQCA). Applied Social Research Methods Series, 51(1952), 33-69. doi:10.4135/9781452226569

Rihoux, B., \& Ragin, C. C. (Eds.). (2009). Configurational comparative method: Qualitative comparative analysis and related techniques. Los Angeles, USA: Sage Publications.

Robbins, S. P., \& Judge, I. A. (2013). Organizational behavior (15th ed.). New Jersey, USA: Pearson Education, Inc.

Schein, E. H. (1996). Culture: The missing concept in organization studies. Source: Administrative Science Quarterly, 41(2), 229240. doi: $10.2307 / 2393715$

Schneider, C. Q., \& Wagemann, C. (2010). Standards of good practice in qualitative comparative analysis (QCA) and fuzzysets. Comparative Sociology, 9(3), 397-418. doi: 10.1163/1569 $13210 X_{12493538729793}$
Siegler, J., Biazzin, C., \& Fernandes, A. R. (2014). Fragmentação do conhecimento científico em administração: Uma análise crítica. RAE-Revista de Administração de Empresas, 54(3), 254-267. doi: 10.1590/So034-759020140302

Silva, I. B., \& Nakano, T. de C. (2011). Modelo dos cinco grandes fatores da personalidade: Análise de pesquisas. Avaliação Psicológica, 10(1), 51-62. Recuperado de http://pepsic. bvsalud.org/scielo.php?script=sci_arttext\&pid $=$ S167704712011000100006\&lng=pt\&nrm=iso\&tlng=pt

Sistema de Avaliação de Testes Psicológicos. (2016). SATEPSI. Retrieved from http://satepsi.cfp.org.br/default.cfm

Souza, E. C. L. De, \& Fenili, R. R. (2016). O estudo da cultura organizacional por meio das práticas: Uma proposta à luz do legado de Bourdieu/The study of organizational culture through practices: A proposal in the light of Bourdieu's legacy. Caderno EBAPE.BR, 14(4), 872-890. doi: 10.1590/1679-395141183

Stank, T. P., Dittmann, J. P., \& Autry, C. W. (2011). The new supply chain agenda: A synopsis and directions for future research. Journal of Small Business and Enterprise Development, 12(4), 564-578. doi: 10.1108/02656710210415703

Stone, H., Bleibaum, R. N., \& Thomas, H. A. (2012). Descriptive Analysis. In H. Stone, R. N. Bleibaum, \& H. A. Thomas (Eds.), Sensory Evaluation Practices (4th ed., pp. 201-245). New York: Elsevier Inc. doi: 10.1016/C2009-0-63404-8

Thaler, R. H. (2016). "Behavioral Economics: Past, Present, and Future." American Economic Review, 106 (7): 1577-1600.

Tatham, P., Wu, Y., Kovács, G., \& Butcher, T. (2017). Supply chain management skills to sense and seize opportunities. The International Journal of Logistics Management, 28(2), 266289. doi: 10.1108/IJLM-04-2014-0066

Touboulic, A., \& Walker, H. (2015). Love me, love me not: A nuanced view on collaboration in sustainable supply chains. Journal of Purchasing and Supply Management, 21(3), 178191. doi: 10.1016/j.pursup.2015.05.001

Tsanos, C. S., Zografos, K. G., \& Harrison, A. (2014). Developing a conceptual model for examining the supply chain relationships between behavioural antecedents of collaboration, integration and performance. The International Journal of Logistics Management, 25(3), 418-462. doi: 10.1108/IJLM-02-2012-0005

Wang, L. (2016). The internal and external resource integration in operation process: A case study. Journal of Service Science and Management, 9, 125-133.

\section{CONTRIBUIÇÃO DOS AUTORES}

Roberta de Cássia Macedo, Ricardo Silveira Martins e Jonathan Simões Freitas trabalharam na conceitualização e abordagem teórica-metodológica. A revisão teórica foi conduzida pela Roberta de Cássia Macedo. A coleta de dados foi coordenada pelo(a) Ricardo Silveira Martins e executada por Roberta de Cássia Macedo. Participaram da análise de dados Roberta de Cássia Macedo, Ricardo Silveira Martins e Jonathan Simões Freitas. Todos os autores participaram da redação e revisão final do manuscrito. 